\title{
Weber-Type Integral Transform Connected with Robin-Type Boundary Conditions
}

\author{
Thanaa Elnaqeeb ${ }^{1,2}$, Nehad Ali Shah ${ }^{3,4, *}$ and Dumitru Vieru ${ }^{5}$ \\ 1 Department of Mathematics and Statistics, Faculty of Science, Taif University, Taif 888, Saudi Arabia; \\ thanaaa_1@yahoo.com \\ 2 Department of Mathematics, Faculty of Science, Zagazig University, Zagazig 44519, Egypt \\ 3 Informetrics Research Group, Ton Duc Thang University, Ho Chi Minh City 58307, Vietnam \\ 4 Faculty of Mathematics \& Statistics, Ton Duc Thang University, Ho Chi Minh City 58307, Vietnam \\ 5 Department of Theoretical Mechanics, Technical University, “Gheorghe Asachi” of Iasi, 6600 Iasi, Romania; \\ dumitru.vieru@tuiasi.ro \\ * Correspondence: nehad.ali.shah@tdtu.edu.vn
}

Received: 12 July 2020; Accepted: 5 August 2020; Published: 11 August 2020

\begin{abstract}
A new Weber-type integral transform and its inverse are defined for the representation of a function $f(r, t),(r, t) \in[R, 1] \times[0, \infty)$ that satisfies the Dirichlet and Robin-type boundary conditions $f(R, t)=f_{1}(t), f(1, t)-\left.\alpha \frac{\partial f(r, t)}{\partial r}\right|_{r=1}=f_{2}(t)$, respectively. The orthogonality relations of the transform kernel are derived by using the properties of Bessel functions. The new Weber integral transform of some particular functions is determined. The integral transform defined in the present paper is a suitable tool for determining analytical solutions of transport problems with sliding phenomena that often occur in flows through micro channels, pipes or blood vessels. The heat conduction in an annular domain with Robin-type boundary conditions is studied. The subroutine "root $(\cdot)$ " of the Mathcad software is used to determine the positive roots of the transcendental equation involved in the definition of the new integral transform.
\end{abstract}

Keywords: integral transforms; Weber transform; Bessel functions

\section{Introduction}

Integral transform methods are powerful analytical tools in the solving of mixed boundary value problems. The transform methods provide a way of reducing of the complexity of solving problems and allow for obtaining analytical or semi-analytical solutions. Additionally, integral transforms lead to a reduction in the dimensionality of the problems and, in the hybrid numerical techniques, can significantly reduce the computation cost. Watson [1] has studied Weber's integral transforms for circular cylinder functions with Dirichlet and Neumann boundary conditions.

Thambynayagam and Habashy [2] have studied the Weber-type transform pair for the representation of a function $f(r), r \in[a, \infty)$ which satisfies the Robin-type boundary condition $f(a)+b f^{\prime}(a)=0$. Battig and Kalla [3] have used the Weber's transform for the study of heat conduction through an infinite medium with a cylindrical cavity.

Zhang and Tong [4] defined a generalized Weber transform and its inverse formula for functions defined on the domain $[a, \infty)$. The definition contains a Weber transform as a particular case and, as an application, they used the generalized Weber transform to study a well with constant production in an infinite fractal fluid reservoir in a porous medium.

A generalization of a Neumann-Weber integral transform for complex Bessel indices has been proposed by Buschle et al. [5]. The new integral transform was successfully applied for solving diffusion equations and heat equations around cylindrical bodies. 
Gorshkov [6] determined the explicit formula for the solution of the unsteady Stokes system in the exterior of a disc with a non-slip condition and a given velocity at infinity. By using the vorticity operator, the author transformed the Stokes system into a heat equation with zero Robin-type conditions on the disc boundary. Then a Weber-Orr integral transform was used to solve these equations.

A generalized integral transform with Weber's parabolic cylinder function for a class of generalized functions was defined by Mahato and Saksena [7]. As a particular case, they found that this integral transform reduces to a Laplace transform.

Colzani et al. [8] have proved the asymptotic decay of some integral transforms, namely, the sine/cosine Fourier transform, Laplace transform and Hankel transform. They have shown that if the original function is asymptotic, then its transform is asymptotic to infinity.

The differentiation properties of the fractional Hankel transform of functions that involves the higher-order derivative have been studied by Jafar et al. [9]. The obtained results are important because they could be a suitable tool for solving differential equations of higher orders. An interesting application of the Shehu transformation, defined by Maitama and Zhao [10], has been investigated by Khan et al. [11]. They obtained the approximate analytical solution for a two-dimensional time-fractional differential equation.

Kabal and Cassiani [12] have introduced the Weber-Goldstein integral transform and have studied its application for solving boundary value problems in well hydraulics. Kellendonk and Richard [13] studied the properties of a Weber-Schafheitlin integral containing Hankel functions of the first kind.

Some integral transforms involving a product of Humbert and Bessel functions with an exponential weight have been studied by Belafhal et al. [14]. They have evaluated the proposed integral transforms in terms of hypergeometric functions. Additionally, some transformation formulae are expressed in terms of Appell functions. As an application, the authors have investigated the generalized Humbert-Gaussian beams propagating in space.

Chen and Liang [15] studied the higher-order numerical quadrature for the integration of systems containing Bessel, Anger and Weber functions. The authors have obtained approximate solutions for the differential equations and truncating the asymptotic series. The efficiency of the method was demonstrated by numerical examples.

The new index integral transform with Weber-type kernels consisting of products of Bessel functions of the first and second kind have been studied by Yakubovich [16]. The mapping properties and inversion formulas were established in Lebesgue spaces. The obtained results wereapplied to solve the boundary value problems on the wedge for a fourth-order partial differential equation.

Aghili [17] has determined the analytical solutions for the time-fractional diffusion problem with mixed boundary conditions by using the operational method. Al-Musallam and Tuan [18] derived a modified Weber transform and its reciprocal, the index Weber transforms, and have given the space of functions on which they act.

A noteworthy advancement was made in the investigation of laminar progressions of Newtonian fluids over complex surfaces. The numerical simulation of such flows is greatly simplified if the complex boundary surface and microscopic boundary conditions are approximated by a smooth boundary and an effective boundary condition. Researchers reviewed physical experiments and computational studies conducted to deduce effective boundary conditions for flows over rough or structured surfaces, surfaces with chemical patterns, nano-bubbles or polymer layers and super hydrophobic surfaces. In many of these situations, the proposed effective boundary condition was a partial slip boundary condition $[19,20]$.

In many practical applications, such as fluid flow models in an annular channel between two coaxial circular cylinders, the Weber transform coupled with the Laplace transform can be used to obtain analytical or semi-analytical solutions.

To flows where the boundary conditions are of a Dirichlet or Neumann type, the Weber transform can be successfully applied. If, on one of the cylindrical surfaces, the boundary condition is of 
the Robin-type, then the classical form of the Weber transform cannot lead to the analytical solution of such a problem.

In the present paper, we introduce a new Weber-type transform pair for the representation of a differentiable function $f(r, t),(r, t) \in[R, 1] \times[0, \infty)$ that satisfies the Dirichlet condition $f(R, t)=h_{1}(t)$ and the Robin condition $f(1, t)-\left.\alpha \frac{\partial f(r, t)}{\partial r}\right|_{r=1}=h_{2}(t)$, respectively.

The integral transform defined in this paper is a useful tool for determining the analytical solutions of the transport processes in which the slip phenomena are present.

The new Weber's transform for some particular functions is determined. The heat conduction process in an annular medium with a Robin-type boundary condition is also studied. Even if this problem is studied by many authors, to our knowledge, there are no analytical solutions for such a diffusion problem. The definition of the new Weber transform involves the positive roots of a transcendental equation with Bessel functions. These roots are determined by using the subroutine "root $(\cdot)$ " of the Mathcad software.

\section{Introductory Definitions and Properties}

The Bessel differential equation and its solution are well defined in many books $[8,9]$. We introduce the following Bessel differential equation:

$$
z^{2} \frac{d^{2} y(z)}{d z^{2}}+z \frac{d y(z)}{d z}+\left(z^{2}-p^{2}\right) y(z)=0, p \in \mathbb{C}
$$

The solution of the Bessel equation is given by Bessel functions [21,22]. The Bessel function of order $p$ and of the first kind is defined as

$$
J_{p}(z)=\left(\frac{z}{2}\right)^{p} \sum_{m=0}^{\infty} \frac{\left(-z^{2} / 4\right)^{m}}{m ! \Gamma(m+p+1)}
$$

and the Bessel function of order $p$ and the second kind satisfies the relationship

$$
W\left[J_{p}(z), Y_{p}(z)\right]=\operatorname{det}\left(\begin{array}{cc}
J_{p}(z) & Y_{p}(z) \\
J_{p}^{\prime}(z) & Y_{p}^{\prime}(z)
\end{array}\right)=\frac{2}{\pi z} .
$$

The Bessel function and its properties are given in detail and illustrated and in many books [1,23]. In addition, Bessel functions satisfy the following recurrence formulas

$$
\begin{gathered}
Z_{p-1}(z)-\frac{2 p}{z} Z_{p}(z)+Z_{p+1}(z)=0 \\
Z_{p-1}(z)-Z_{p+1}(z)=2 Z_{p}^{\prime}(z) \\
Z_{p}^{\prime}(z)=Z_{p-1}(z)-\frac{p}{z} Z_{p}(z) \\
Z_{p}^{\prime}(z)=-Z_{p+1}(z)+\frac{p}{z} Z_{p}(z)
\end{gathered}
$$

where $Z_{p}(z)$ can be $J_{p}(z)$ or $Y_{p}(z)$.

\section{New Weber-Type Integral Transform}

If the function $f(r, t)$ is a differentiable function for $(r, t) \in[R, 1] \times[0, \infty), R>0$, then the Weber transform is defined as

$$
\hat{f}\left(r_{n}, t\right)=\int_{R}^{1} f(r, t) r Z_{00}\left(r, r_{n}\right) d r .
$$

where

$$
Z_{00}\left(r, r_{n}\right)=J_{0}\left(r r_{n}\right) Y_{0}\left(R r_{n}\right)-J_{0}\left(R r_{n}\right) Y_{0}\left(r r_{n}\right)
$$


and $r_{n}, n=1,2, \ldots$ are the positive roots of the transcendental equation.

$$
Z_{00}\left(1, r_{n}\right)-\left.\alpha \frac{d Z_{00}\left(r, r_{n}\right)}{d r}\right|_{r=1}=0, \alpha \in \mathbb{R}
$$

Since

$$
\begin{aligned}
& \frac{d Z_{00}\left(r, r_{n}\right)}{d r}=-r_{n} Z_{10}\left(r, r_{n}\right), \\
& Z_{10}\left(r, r_{n}\right)=J_{1}\left(r r_{n}\right) Y_{0}\left(R r_{n}\right)-J_{0}\left(R r_{n}\right) Y_{1}\left(r r_{n}\right),
\end{aligned}
$$

Equation (7) becomes equivalent to the equation

$$
\mathrm{Z}_{00}\left(1, r_{n}\right)+\alpha r_{n} \mathrm{Z}_{10}\left(1, r_{n}\right)=0
$$

By integrating by parts and using the property of the Bessel functions $\int z^{n} J_{n-1}(z)=z^{n} J_{n}(z)+C, n \in \mathbb{N}$, then the following propositions can be proved:

Proposition 1. For $k \neq n$,

(a)

$$
\begin{aligned}
& A_{1}(k, n)=\int_{R}^{1} r J_{0}\left(r r_{k}\right) J_{0}\left(r r_{n}\right) d r= \\
& \left(r_{k}^{2}-r_{n}^{2}\right)^{-1}\left[r_{k} J_{1}\left(r_{k}\right) J_{0}\left(r_{n}\right)-r_{n} J_{1}\left(r_{n}\right) J_{0}\left(r_{k}\right)-R r_{k} J_{1}\left(R r_{k}\right) J_{0}\left(R r_{n}\right)+R r_{n} J_{1}\left(R r_{n}\right) J_{0}\left(R r_{k}\right)\right]
\end{aligned}
$$

(b)

$$
\begin{aligned}
& A_{2}(k, n)=\int_{R}^{1} r J_{0}\left(r r_{k}\right) Y_{0}\left(r r_{n}\right) d r= \\
& \left(r_{k}^{2}-r_{n}^{2}\right)^{-1}\left[r_{k} J_{1}\left(r_{k}\right) Y_{0}\left(r_{n}\right)-r_{n} Y_{1}\left(r_{n}\right) J_{0}\left(r_{k}\right)-R r_{k} J_{1}\left(R r_{k}\right) Y_{0}\left(R r_{n}\right)+R r_{n} Y_{1}\left(R r_{n}\right) J_{0}\left(R r_{k}\right)\right]
\end{aligned}
$$

(c)

$$
\begin{aligned}
& A_{3}(k, n)=\int_{R}^{1} r J_{0}\left(r r_{n}\right) Y_{0}\left(r r_{k}\right) d r= \\
& \left(r_{k}^{2}-r_{n}^{2}\right)^{-1}\left[r_{k} Y_{1}\left(r_{k}\right) J_{0}\left(r_{n}\right)-r_{n} J_{1}\left(r_{n}\right) Y_{0}\left(r_{k}\right)-R r_{k} Y_{1}\left(R r_{k}\right) J_{0}\left(R r_{n}\right)+R r_{n} J_{1}\left(R r_{n}\right) Y_{0}\left(R r_{k}\right)\right]
\end{aligned}
$$

(d)

$$
\begin{aligned}
& A_{4}(k, n)=\int_{R}^{1} r Y_{0}\left(r r_{k}\right) Y_{0}\left(r r_{n}\right) d r= \\
& \left(r_{k}^{2}-r_{n}^{2}\right)^{-1}\left[r_{k} Y_{1}\left(r_{k}\right) Y_{0}\left(r_{n}\right)-r_{n} Y_{1}\left(r_{n}\right) Y_{0}\left(r_{k}\right)-R r_{k} Y_{1}\left(R r_{k}\right) Y_{0}\left(R r_{n}\right)+R r_{n} Y_{1}\left(R r_{n}\right) Y_{0}\left(R r_{k}\right)\right]
\end{aligned}
$$

Proposition 2. The following relations are true:

(a)

(b)

$$
\begin{aligned}
& B_{1}(n, n)=\int_{R}^{1} r J_{0}\left(r r_{n}\right) J_{0}\left(r r_{n}\right) d r= \\
& \frac{1}{2}\left[J_{0}^{2}\left(r_{n}\right)+J_{1}^{2}\left(r_{n}\right)-R^{2} J_{0}^{2}\left(R r_{n}\right)-R^{2} J_{1}^{2}\left(R r_{n}\right)\right]
\end{aligned}
$$

$$
\begin{aligned}
& B_{2}(n, n)=\int_{R}^{1} r J_{0}\left(r r_{n}\right) Y_{0}\left(r r_{n}\right) d r= \\
& \frac{1}{2}\left[J_{0}\left(r_{n}\right) Y_{0}\left(r_{n}\right)+J_{1}\left(r_{n}\right) Y_{1}\left(r_{n}\right)-R^{2} J_{0}\left(R r_{n}\right) Y_{0}\left(R r_{n}\right)-R^{2} J_{1}\left(R r_{n}\right) Y_{1}\left(R r_{n}\right)\right]
\end{aligned}
$$


(c)

$$
\begin{aligned}
& B_{3}(n, n)=\int_{R}^{1} r Y_{0}\left(r r_{n}\right) Y_{0}\left(r r_{n}\right) d r= \\
& \frac{1}{2}\left[Y_{0}^{2}\left(r_{n}\right)+Y_{1}^{2}\left(r_{n}\right)-R^{2} Y_{0}^{2}\left(R r_{n}\right)-R^{2} Y_{1}^{2}\left(R r_{n}\right)\right]
\end{aligned}
$$

By using the transcendental Equation (9) of roots $r_{n}$, and the formulas which are given by Propositions 1 and 2, then the following results regarding the orthogonality of functions $Z_{00}\left(r, r_{n}\right)$ can be obtained.

Proposition 3. Eigen functions $Z_{00}\left(r, r_{n}\right)$ satisfy the following orthogonal relations:

$$
\begin{aligned}
& I_{k n}=\int_{R}^{1} r Z_{00}\left(r, r_{k}\right) Z_{00}\left(r, r_{n}\right) d r=0, k \neq n, \\
& I_{n n}=\int_{R}^{1} r Z_{00}\left(r, r_{n}\right) Z_{00}\left(r, r_{n}\right) d r=\frac{\pi^{2}\left(1+\alpha^{2} r_{n}^{2}\right) Z_{00}^{2}\left(1, r_{n}\right)-4 \alpha^{2}}{2 \alpha^{2} \pi^{2} r_{n}^{2}} .
\end{aligned}
$$

Proposition 4. The inversion formula.

The inverse Weber transform is given by

$$
f(r, t)=\sum_{n=1}^{\infty} \frac{Z_{00}\left(r, r_{n}\right)}{I_{n n}} \hat{f}\left(r_{n}, t\right)
$$

Proof. Consider that the function $f(r, t)$ can be written in the series form as

$$
f(r, t)=\sum_{k=1}^{\infty} C\left(r_{k}, t\right) Z_{00}\left(r, r_{k}\right)
$$

By multiplying Equation (19) with $r Z_{00}\left(r, r_{n}\right)$, integrating with respect to $r$ from $R$ to 1 and by using the relations given in Proposition 3 , we find the following expression for the coefficients $C\left(r_{n}, t\right)$

$$
C\left(r_{n}, t\right)=\frac{\hat{f}\left(r_{n}, t\right)}{I_{n n}}
$$

By substituting Equation (20) into Equation (19), the inverse formula given in Equation (18) can be obtained.

\section{Example}

In this section, we apply the above results to find the Weber transform of the following function

$$
f(r)=\frac{R^{2}-r^{2}}{R^{2}+2 \alpha-1}, r \in[R, 1] .
$$


By using the new Weber transform given by Equation (5) and by the help of the properties of Bessel functions, then, after straightforward calculus, one can obtain the transform of the function given in Equation (21) as

$$
\begin{aligned}
& \hat{f}\left(r_{n}\right)=\frac{\hat{g}\left(r_{n}\right)}{R^{2}+2 \alpha-1}, \\
& \hat{g}\left(r_{n}\right)=\frac{-R^{2}}{\alpha r_{n}^{2}} Z_{00}\left(1, r_{n}\right)-\frac{2 R^{2}}{\pi r_{n}^{2}}+\frac{(2 \alpha-1) r_{n}^{2}+4}{r_{n}^{3}} Z_{10}\left(1, r_{n}\right)+\frac{2\left(R^{2} r_{n}^{2}-4\right)}{\pi r_{n}^{4}}
\end{aligned}
$$

Then, by using the inversion formula given in Equation (18), we can obtain the following representation

$$
\frac{R^{2}-r^{2}}{R^{2}+2 \alpha-1}=\frac{1}{R^{2}+2 \alpha-1} \sum_{n=1}^{\infty} \frac{Z_{00}\left(r, r_{n}\right)}{I_{n n}} \hat{g}\left(r_{n}\right), r \in[R, 1) .
$$

To use the new Weber transform, the roots of the transcendental Equation (9) must be known. Based on the following approximations of Bessel functions

$$
J_{m}(z) \cong \sqrt{\frac{2}{\pi z}} \cos \left(z-\frac{(2 m+1) \pi}{4}\right), Y_{m}(z) \cong \sqrt{\frac{2}{\pi z}} \sin \left(z-\frac{(2 m+1) \pi}{4}\right), z \gg\left|m^{2}-1 / 4\right|,
$$

it is easy to see that Equation (9) has a unique root $r_{n}$ in the interval $\left(\frac{(2 n-1) \pi}{2(1-R)}, \frac{(2 n+1) \pi}{2(1-R)}\right), n=1,2, \ldots$. Now, by using the subroutine $\operatorname{root}\left(f(r), r, a_{n}, b_{n}\right) a_{n}=\frac{(2 n-1) \pi}{2(1-R)}, b_{n}=\frac{(2 n+1) \pi}{2(1-R)}$, in the Mathcad 15 software, the roots of Equation (9) can be generated. For $R=0.2, \alpha=0.5$, the first five roots of Equation (9) are $r_{1}=5.246, r_{2}=9.436, r_{3}=13.472, r_{4}=17.460, r_{5}=21.425$. It is easy to check that the function $f(r)$ defined by Equation (21) satisfies the two boundary conditions $f(R)=0, f(1)-\left.\alpha \frac{\partial f(r)}{\partial r}\right|_{r=1}=1$.

The profiles of the exact and approximate values of the function $f(r)$ which, given by Equation (23), are presented in Figure 1 for $R=0.2, \alpha=0.001$ and $r \in[0.2,0.99]$. In the sum of Equation (23), 1000 terms were considered. As shown in Figure 1, a very good agreement between the exact and approximate values has been obtained.

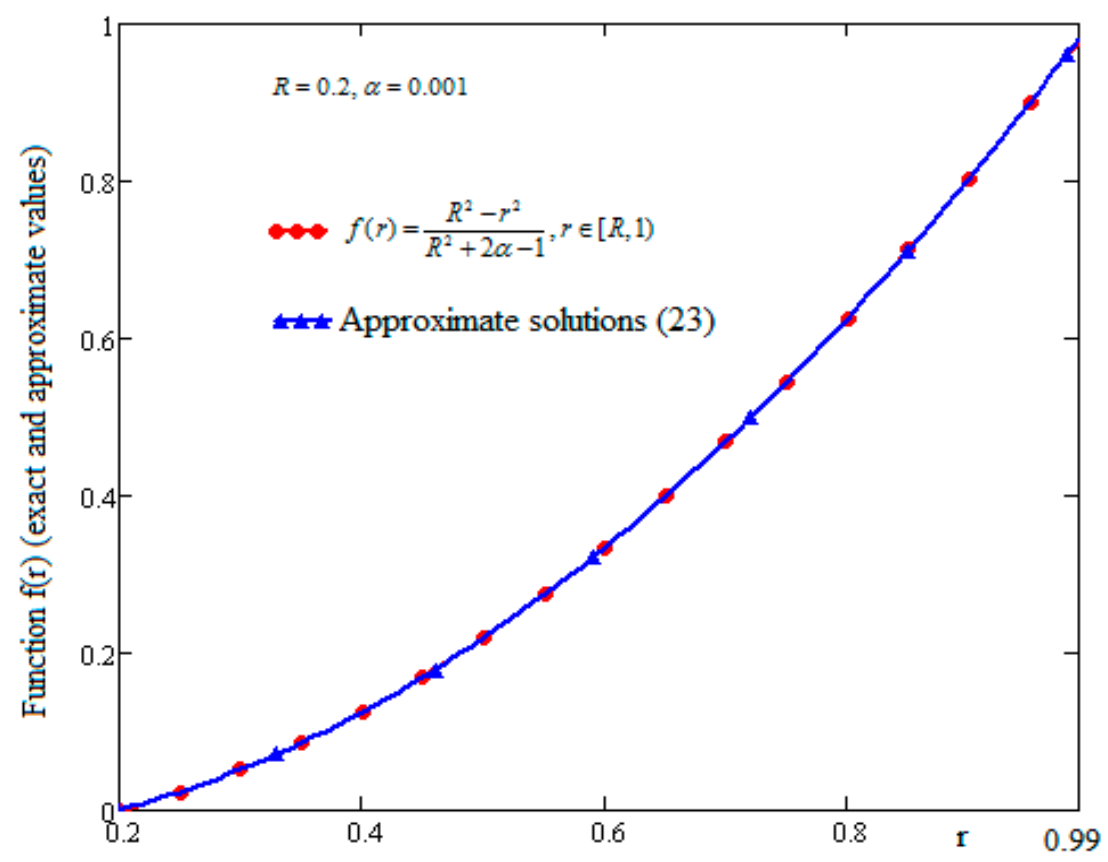

Figure 1. The profiles of function given by Equation (23). 


\section{Application to the Transient Heat Conduction in an Annular Domain}

In this section, an application of the heat conduction in an annular domain is examined. Consider that the radii of two co-axial circular cylinders are $R_{1}, R_{2}, R_{1}<R_{2}$. The heat equation for the axisymmetric case in the domain between these two cylinders in cylindrical coordinates is given by [24,25]:

$$
\rho c_{p} \frac{\partial T_{1}\left(r_{1}, t_{1}\right)}{\partial t_{1}}=k \frac{1}{r_{1}} \frac{\partial}{\partial r_{1}}\left(r_{1} \frac{\partial T_{1}\left(r_{1}, t_{1}\right)}{\partial r_{1}}\right),
$$

where $T_{1}\left(r_{1}, t_{1}\right)$ denotes the dimensional temperature of the medium, $r_{1} \in\left[R_{1}, R_{2}\right]$ is the radial coordinate, $t_{1}$ is the time, $\rho$ is the density of the medium, $c_{p}$ is the specific heat and $k$ is the thermal conductivity of the medium.

Using the non-dimensional parameters

$$
r=\frac{r_{1}}{R_{2}}, t=\frac{\lambda t_{1}}{R_{2}^{2}}, \lambda=\frac{k}{\rho c_{p}}, T=\frac{T_{1}}{T_{0}}, R=\frac{R_{1}}{R_{2}},
$$

where $T_{0}$ is a characteristic temperature, we obtain the following dimensionless heat equation:

$$
\frac{\partial T(r, t)}{\partial t}=\frac{1}{r} \frac{\partial}{\partial r}\left(r \frac{\partial T(r, t)}{\partial r}\right),(r, t) \in[R, 1] \times[0, \infty) .
$$

Along with heat Equation (27), we consider the initial and boundary conditions as follows

$$
\begin{gathered}
T(r, 0)=0, r \in[R, 1], \\
T(R, t)=0, \\
T(1, t)-\left.\alpha \frac{\partial T(r, t)}{\partial r}\right|_{r=1}=f_{0}(t),
\end{gathered}
$$

where $f_{0}(t)$ is a continuous piecewise function of an exponential order to infinity and $f_{0}(0)=0$.

To find an analytical solution for Equation (27) subjected to the initial and boundary conditions given in Equations (28) and (29), we use the Laplace transform and the newly defined form of the Weber transform given in Section 3.

By applying the Laplace transform technique to Equation (27) and by using the initial condition given in Equation (28), one can obtain the following transformed equation

$$
s \bar{T}(r, s)=\frac{1}{r} \frac{\partial}{\partial r}\left(r \frac{\partial \bar{T}(r, s)}{\partial r}\right)
$$

where $\bar{T}(r, s)=\int_{0}^{\infty} T(r, t) \exp (-s t) d t$ is the Laplace transform of the function $T(r, t)$ and $s$ denotes the transform parameter. The function $\bar{T}(r, s)$ has to satisfy the boundary conditions

$$
\begin{aligned}
& \bar{T}(R, t)=0, \\
& \bar{T}(1, t)-\left.\alpha \frac{\partial \bar{T}(r, s}{\partial r}\right|_{r=1}=\bar{f}_{0}(s) .
\end{aligned}
$$

By using definition (5), the integration by parts and the properties of Bessel functions, we obtain

$$
\begin{gathered}
\int_{R}^{1} \frac{1}{r} \frac{\partial}{\partial r}\left(r \frac{\partial \bar{T}(r, s)}{\partial r}\right) r Z_{00}\left(r, r_{n}\right) d r=-r_{n}^{2} \hat{\bar{T}}\left(r_{n}, s\right)-\frac{2}{\pi} \bar{T}(R, s)+ \\
{\left[\bar{T}(1, s)-\left.\alpha \frac{\partial \bar{T}(r, s)}{\partial r}\right|_{r=1}\right] r_{n} Z_{10}\left(1, r_{n}\right)}
\end{gathered}
$$


By applying the Weber transform to Equation (30) and using Equation (32), we have

$$
s \hat{\bar{T}}\left(r_{n}, s\right)=-r_{n}^{2} \hat{\bar{T}}\left(r_{n}, s\right)-\frac{2}{\pi} \bar{T}(R, s)+\left[\bar{T}(1, s)-\left.\alpha \frac{\partial \bar{T}(r, s)}{\partial r}\right|_{r=1}\right] r_{n} Z_{10}\left(1, r_{n}\right) .
$$

By using the boundary conditions (31), we obtain

$$
\hat{\bar{T}}\left(r_{n}, s\right)=\frac{\bar{f}_{0}(s)}{s+r_{n}^{2}} r_{n} Z_{10}\left(1, r_{n}\right),
$$

respectively, the equivalent form

$$
\hat{\bar{T}}\left(r_{n}, s\right)=\hat{f}\left(r_{n}\right) \bar{f}_{0}(s)+\hat{\bar{h}}\left(r_{n}, s\right) \bar{f}_{0}(s),
$$

where $\hat{f}\left(r_{n}\right)$ is given in Equation (22) and $\hat{\bar{h}}\left(r_{n}, s\right)$ is

$$
\begin{aligned}
& \hat{\bar{h}}\left(r_{n}, s\right)=\frac{4-\left(R^{2}+2 \alpha-1\right) r_{n}^{2}}{r_{n}^{3}\left(R^{2}+2 \alpha-1\right)} \frac{\bar{f}_{0}(s)}{s+r_{n}^{2}}+\frac{4}{r_{n}\left(R^{2}+2 \alpha-1\right)} \frac{\bar{f}_{0}(s)}{s+r_{n}^{2}}+ \\
& \quad \frac{2(\pi-1) R^{2} r_{n}^{2}+8}{\pi r_{n}^{4}\left(R^{2}+2 \alpha-1\right)} \bar{f}_{0}(s) .
\end{aligned}
$$

Thus, the inverse Laplace transform of the function $\hat{\bar{h}}\left(r_{n}, s\right)$ is given by

$$
\begin{aligned}
\hat{h}\left(r_{n}, t\right)= & \frac{4-\left(R^{2}+2 \alpha-1\right) r_{n}^{2}}{r_{n}^{3}\left(R^{2}+2 \alpha-1\right)} \int_{0}^{t} f^{\prime}{ }_{0}(t-\tau) e^{-r_{n}^{2} \tau} d \tau+\frac{4}{r_{n}\left(R^{2}+2 \alpha-1\right)} \int_{0}^{t} f_{0}(t-\tau) e^{-r_{n}^{2} \tau} d \tau+ \\
& \frac{2(\pi-1) R^{2} r_{n}^{2}+8}{\pi r_{n}^{4}\left(R^{2}+2 \alpha-1\right)} f_{0}(t) .
\end{aligned}
$$

By applying the inverse Laplace transform and Weber transform to Equation (35), one can get the temperature solution as the following

$$
T(r, t)=\frac{R^{2}-r^{2}}{R^{2}+2 \alpha-1} f_{0}(t)+\sum_{n=1}^{\infty} \frac{Z_{00}\left(r, r_{n}\right)}{I_{n n}} \hat{h}\left(r_{n} t\right) .
$$

One can check that the functions $f(r)$ and $Z_{00}\left(r, r_{n}\right)$ satisfy the following conditions $f(R)=0$, $f(1)-\left.\alpha \frac{d f(r)}{d r}\right|_{r=1}=1$, respectively, $Z_{00}\left(R, r_{n}\right)=0, Z_{00}\left(1, r_{n}\right)-\left.\alpha \frac{d Z_{00}\left(r, r_{n}\right)}{d r}\right|_{r=1}=0$. Therefore, the function $T(r, t)$ satisfies the boundary conditions introduced in Equation (31).

\section{Conclusions}

A new Weber-type transform pair for the representation of a differentiable function $f(r, t)$, $(r, t) \in[R, 1] \times[0, \infty)$ and that satisfies the Dirichlet condition $f(R, t)=h_{1}(t)$, and the Robin condition $f(1, t)-\left.\alpha \frac{\partial f(r, t)}{\partial r}\right|_{r=1}=h_{2}(t)$, respectively, has been defined.

The orthogonality relations of the integral transform's kernel are derived by using the properties of Bessel functions. Based on the orthogonality relations of Weber's kernel, the inversion formula of the integral transform has been determined.

The new Weber integral transform of some particular functions has been determined.

As an application, the heat conduction process in an annular medium with a Robin-type boundary condition has been investigated; the analytical solution of the proposed problem has been determined. Such a solution is new in the literature.

The integral transform defined in the present paper is a suitable tool for determining analytical solutions of the transport problems with sliding phenomena that often occur in flows through micro channels, pipes or blood vessels. 
The positive roots of the transcendental equation with Bessel functions, used in the integral transform's definition, have been determined by using a subroutine of the Mathcad software.

Author Contributions: Conceptualization, D.V. and N.A.S.; methodology, D.V.; software, T.E.; validation, T.E., N.A.S. and D.V.; formal analysis, D.V.; investigation, T.E.; writing-original draft preparation, N.A.S.; writing-review and editing, T.E.; visualization and supervision, D.V.; project administration, N.A.S. All authors have read and agreed to the published version of the manuscript.

Funding: This research received no external funding.

Conflicts of Interest: The authors declare no conflict of interest.

\section{References}

1. Watson, G.N. Theory of Bessel Functions; Cambridge University Press: Cambridge, UK, 1980.

2. Thambynayagam, R.K.M.; Habashy, T.M. A new Weber-type transform. Q. Appl. Math. 2003, 61, 485-493. [CrossRef]

3. Battig, A.; Kalla, S.L. Weber transform in a thermal conduction problem. Rev. Bras. Phys. 1983, 13, $753-756$.

4. Zhang, X.; Thong, D. A generalized Weber transform and its inverse formula. Appl. Math. Comput. 2007, 193, 116-126. [CrossRef]

5. Buschle, L.R.; Kurz, F.T.; Schlemmer, H.P.; Ziener, C.H. Neumann-Weber integral transform for complex indices. J. Math. Phys. 2019, 60, 043502. [CrossRef]

6. Gorshkov, A.V. Associate Weber-Orr transform, Biot-Savart law and explicit form of the solution of 2D Stokes system in exterior of the disc. J. Math. Fluid Mech. 2019, 21, 41. [CrossRef]

7. Mahato, A.K.; Saksena, K.M. A generalized Laplace transform of generalized functions. Anal. Math. 1992, 18, 139-151. [CrossRef]

8. Colzania, L.; Fontanaa, L.; Laeng, E. Asymptotic decay of Fourier, Laplace and other integral transforms. J. Math. Anal. Appl. 2020, 483, 123560. [CrossRef]

9. Jafara, S.; Iqbalb, S.; Sarwarc, F. Differentiation property of fractional Hankel transform of a function involving higher order derivatives. Am. Sci. Res. J. Eng. Technol. Sci. 2020, 65, 16-28.

10. Maitama, S.; Zhao, W. New integral transform: Shehu transform a generalization of Sumudu and Laplace transform for solving differential equations. arXiv 2019, arXiv:1904.11370.

11. Khan, H.; Farooq, U.; Shah, R.; Baleanu, D.; Kumam, P.; Arif, M. Analytical solutions of (2+time fractional order) dimensional physical models, using modified decomposition method. Appl. Sci. 2020, 10, 122. [CrossRef]

12. Kabala, Z.J.; Cassiani, G. Well hydraulics with the Weber-Goldstein transforms. Transp. Porous Media 1997, 29, 225-246. [CrossRef]

13. Kellendonk, J.; Richard, S. Weber-Schafheitlin-type integrals with exponent 1. Integral Transform. Spec. Funct. 2009, 20, 147-153. [CrossRef]

14. Belafhal, A.; Nossir, N.; Dalil-Essakali, L.; Usman, T. Integral transforms involving the product of Humbert and Bessel functions and its application. Aims Math. 2019, 5, 1260-1274. [CrossRef]

15. Chen, R.; Liang, X. Asymptotic expansions of Bessel, Anger and Weber transformations. J. Math. Anal. Appl. 2010, 372, 377-389. [CrossRef]

16. Yakubovich, S. Index transforms with Weber-type kernels. Integral Transform. Spec. Funct. 2018. [CrossRef]

17. Aghili, A. Complete solution for the time fractional diffusion problem with mixed boundary conditions by operational method. Appl. Math. Nonlinear Sci. 2020, 1-12. [CrossRef]

18. Al-Musallam, F.; Tuan, V.K. A modified and a finite index Weber transforms. J. Anal. Its Appl. 2002, 21, 315-334.

19. LeRoux, C. Flows of incompressible viscous liquids with an isotropic wall slip. J. Math. Anal. Appl. 2018, 465, 723-730. [CrossRef]

20. Baranovskii, E.S. Steady flows of an Oldroyd fluid with threshold slip. Commun. Pure Appl. Anal. 2019, 18, 735-750. [CrossRef]

21. El-Shahed, M.; Shawkey, M. Generalized finite Hankel transform. Integral Transform. Spec. Funct. 2006, 17, 39-44. [CrossRef] 
22. Piessens, R. The Hankel Transforms. In The Transforms and Applications Handbook, 2nd ed.; Alexander, D.P., Ed.; CRC Press LLC: Boca Raton, FL, USA, 2000; Chapter 9.

23. Gray, A.; Mathews, G.B. A Treatise on Bessel Functions and Their Applications to Physics; Dover Publications: New York, NY, USA, 1966.

24. Singh, S.; Jain, P.K.; Uddin, R. Analytical solution to transient heat conduction in polar coordinates with multiple layer in radial direction. Int. J. Sci. 2008, 47, 261-273. [CrossRef]

25. Lu, X.; Tervola, P.; Viljanen, M. Transient analytical solution to heat conduction in composite circular cylinder. Int. J. Heat Mass Transf. 2006, 49, 341-348. [CrossRef]

(C) 2020 by the authors. Licensee MDPI, Basel, Switzerland. This article is an open access article distributed under the terms and conditions of the Creative Commons Attribution (CC BY) license (http://creativecommons.org/licenses/by/4.0/). 